\title{
Penile necrosis requiring penectomy complicating recto-urethral fistula post prostate cancer external beam radiation and brachytherapy
}

\author{
John Kinahan, MD; FRCSC; Howard Pai, MD, FRCSC; ${ }^{*}$ Mildred Martens, MD, FRCPC; \\ Jason Gray, MD, FRCSC,; Darren Biberdorf, MD,FRCSC;, Alex Mihailovic, MD, FRCSC, PhD,; \\ Iain McAuley, MD, FRCSC*
}

*Division of Urology, Vancouver Island Health Authority, Victoria, BC; 'Department of Radiation Oncology, BC Cancer Agency - Vancouver Island Centre, Victoria, BC; §Division of Laboratory Medicine and

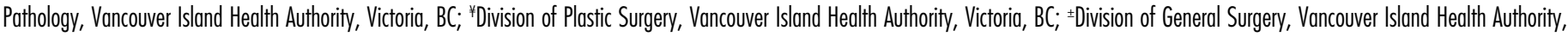
Victoria, BC; £Division of Critical Care, Vancouver Island Health Authority, Victoria, BC

Cite as: Can Urol Assoc J 2014;8(1-2):e57-9. http://dx.doi.org/10.5489/cuaj.1112 Published online January 14, 2014.

\section{Abstract}

Radiation therapy is a well-recognized treatment for unfavourable risk localized prostate cancer. Radiation induced recto-urethral fistulae are known rare complications particularly from brachytherapy. We report a case of a recto-urethral fistula 7 years postexternal beam radiation and $\mathrm{I}-125$ brachytherapy, which was complicated by a severe polymicrobial soft tissue infection. This infection required penectomy and pelvic exenteration with diverting colostomy, Indiana pouch urinary diversion and gracilis myocutaneuos flap closure of the perineum.

\section{Introduction}

External beam radiation (EBRT) combined with prostate brachytherapy (PB) can be used to treat unfavourable risk localized prostate cancer. Radiation-induced recto-urethral fistulae are serious complications that often require major surgery. We present a case of radiation-induced recto-urethral fistula complicated by necrotizing polymicrobial soft tissue infection requiring emergent colostomy, scrotectomy, and penectomy followed by pelvic exenteration with Indiana pouch urinary diversion.

\section{Case report}

This 55-year-old man presented with a stage T2c/T3aNOM0, Gleason score $4+4$ prostate adenocarcinoma involving 8 of 12 cores and a prostate-specific antigen (PSA) 30. He had no ongoing health problems or surgical history, but smoked half a pack per day and had a history of regular heavy alcohol intake.
He received 1 year of androgen deprivation therapy and concurrent radiation on a study protocol. He received 46 Gy in 23 fractions of EBRT to the pelvic region using high energy $18 \mathrm{MV}$ photons, daily 5 days per week. EBRT was planned using computed tomography (CT)-based simulation and 3D-conformal technique. Parallel opposed anterior-posterior and left-right lateral fields were used to encompass the prostate gland, seminal vesicles and pelvic lymph nodes at risk. A 1-cm margin was contoured around the prostate gland to define the planning target volume. The inferior limit of the fields were set $2 \mathrm{~cm}$ below the apex of the prostate gland and the posterior limit was placed at the S2-S3 vertebrae junction to ensure adequate coverage of the pelvic lymph nodes at risk. Fifteen days after completing EBRT, he underwent PB with pre-planned, real-time transrectal ultrasound guided transperineal permanent implantation of $940.33 \mathrm{mCi}$ activity $\mathrm{I}-125$ sealed radioisotope sources to give a planned minimum peripheral dose of 115 Gy with high-dose sparing of the peri-urethral and rectal regions. The general technique has been previously described. ${ }^{1}$ Post-brachytherapy dosimetry to assess quality of implant was completed using a CT scan 4 weeks after procedure. Prostate volume was only 26 cc. The dose to, and volume received by, the prostate gland was within protocol specifications. The volume of rectum receiving the prescription dose (VR100) was $1.72 \mathrm{cc}$. Calculation of dose to the bulbourethral region was not required for protocol quality assurance and not previously known to be a risk factor for severe urinary or bowel toxicity.

He had no significant acute side effects from treatment. Two years later he developed urinary incontinence which worsened over time. Five years after radiation, he had dysuria and pelvic and perineal pain and underwent cystoscopy revealing necrosis in the prostate gland and a non-functioning, fibrotic urethral sphincter. He declined hyperbaric oxygen therapy. At 7 years post-radiation, he developed a recto-urethral fistula and was referred for urgent surgical 
consultation. He did not have any biopsies of his prostate, bladder or rectum prior to developing the fistula. He was seen 7 days later with a 4-day history of severe genital pain and swelling. Necrotic patches on his penis and scrotum were in keeping with a severe soft tissue infection. His CT revealed a recto-urethral fistula $3.5 \mathrm{~cm}$ above the anorectal junction. A 7-cm abscess connected with gas extending the length of the penis, into the scrotum and up the anterior abdominal wall to above the pubic symphysis (Fig. 1).

Vancomycin, clindamycin and piperacillin/tazobactam were administered. He underwent a laparoscopic colostomy, open cystotomy to place a suprapubic catheter and extensive debridement of the genitals. All penile and scrotal skin was dead and removed. The testicles were viable. The glans penis was viable, but the urethra, the corpus spongiosum and corpora cavernosa were necrotic and a penectomy was performed. The anterior wall of the rectum was absent. Cultures grew Group G Streptococcus, E. coli and genital flora. With antibiotics and vasopressors, he rapidly recovered and underwent a pelvic exenteration, bilateral myocutaneous gracilis flap perineal closure and Indiana pouch urinary diversion 8 days later. He was discharged in good medical condition 18 days postoperatively, but was found dead at home 16 days later with cause unknown. The coroner did not request an autopsy.

Pathology revealed extensive necrosis of the proximal urethra, corpus spongiosum, corpora cavernosa and surrounding soft tissues. Preserved portions of the corpus spongiosum showed arterial hyalinization with occlusion of the lumen. Preserved areas of the corpora cavernosa showed increased collagen and fibrosis (Fig. 2) along with moderate to severe fibrointimal hyperplasia and stenosis in the larger arteries (Fig. 3); moreover, he had medial hypertrophy of the smaller vessels with luminal occlusion (Fig. 4).

\section{Discussion}

The incidence of recto-urethral fistulae is about $0.2 \%$ following PB alone. ${ }^{2,3}$ For patients also treated with EBRT, the incidence of high-grade toxicity can be higher than with either therapy alone. ${ }^{4,5}$ Biopsies of the rectum following radiation is a known risk factor for fistula formation, which this patient did not have. ${ }^{5,6} \mathrm{~A}$ high VR100 from PB has been previously reported to increase risk of rectal toxicity. ${ }^{2}$ This patient's volume was $1.72 \mathrm{cc}$. At our institution, the risk of ulceration and other severe rectal complications was $0.8 \%$ with VR100 $<3 \mathrm{cc}$ for patients receiving PB alone to a prescribed dose of 144 Gy. ${ }^{2}$ Pre-existing micro-vascular disease may have been a predisposing factor as this patient was a known smoker.

We report a new complication where the recto-urethral fistula caused severe polymicrobial soft tissue infection and penile necrosis. Radiation therapy is known to cause fibrosis and vascular compromise, including endarteritis obliterans.?

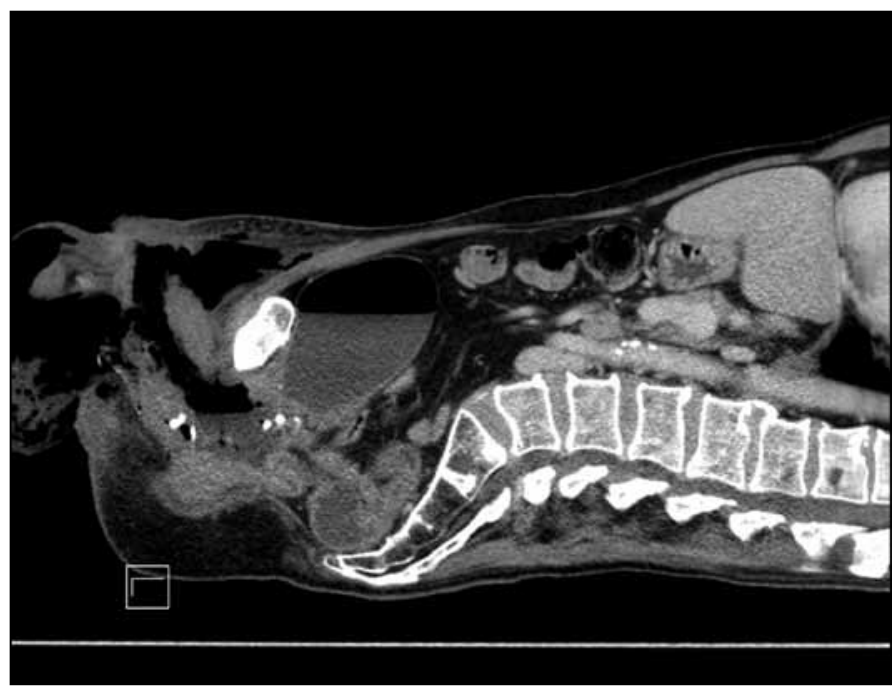

Fig. 1. A defect in the anterior rectal wall communicates with an abscess in the area of the prostate gland. Gas extends along the corpora cavernosa, into the scrotum and into the soft tissue of the abdominal wall above the symphysis pubis.

With EBRT and PB, the bulbourethral region of the penis can receive considerable radiation dose. $^{8}$ In this patient, a portion of the bulbourethra region was included in the high dose region of EBRT and PB; however, the relationship between radiation dose to bulbourethral region and severe urinary and rectal toxicity is not well-described. We postulate that the chronic hyalinization of vessels within the corpora cavernosa and corpus spongiosum resulting from radiation compromised the blood supply within the corporal bodies so that the penis was not spared in this severe soft tissue infection.

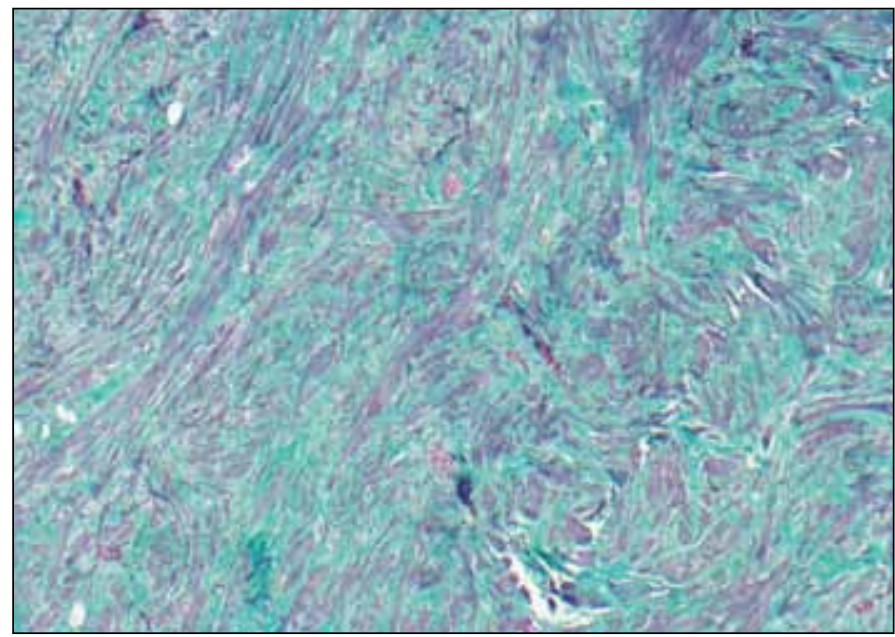

Fig. 2. Corpus cavernosum showing increased collagen (elastic trichrome stain, original magnification 40x). 


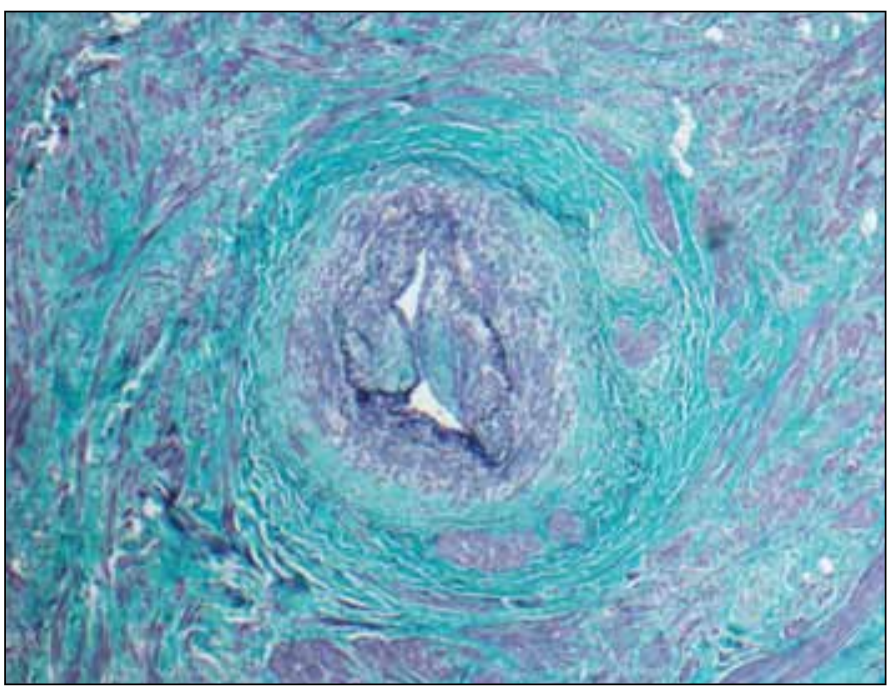

Fig. 3. Moderate to severe fibrointimal thickening and stenosis of large arteries (elastic trichrome stain, original magnification 40x).

\section{Conclusion}

Recto-urethral fistula is a complication of PB. Penile necrosis requiring penectomy is not previously reported as a complication of PB and EBRT for prostate cancer.

Competing interests: Dr. McAuley, Dr. Kinahan, Dr. Pai, Dr. Martens, Dr. Gray, Dr. Biberdorf and Dr. Mihailovic all declare no competing financial or personal interests.

This paper has been peer-reviewed.

\section{References}

1. Blasko JC, Mate T, Sylvester JE, et al. Brachytherapy for carcinoma of the prostate: Techniques, patient selection, and clinical outcomes. Semin Radiat Oncol 2002;12:81-94. http://dx.doi.org/10.1053/ srao.2002.28667

2. Keyes $M$, Spadinger I, Liu $M$, et al. Rectal toxicity and rectal dosimetry in low-dose-rate 1251 permanent prostate implants: A long-term study in 1006 patients. Brachytherapy 2012;11:199-208. http://dx.doi. org/10.1016/i.brachy.2011.05.007

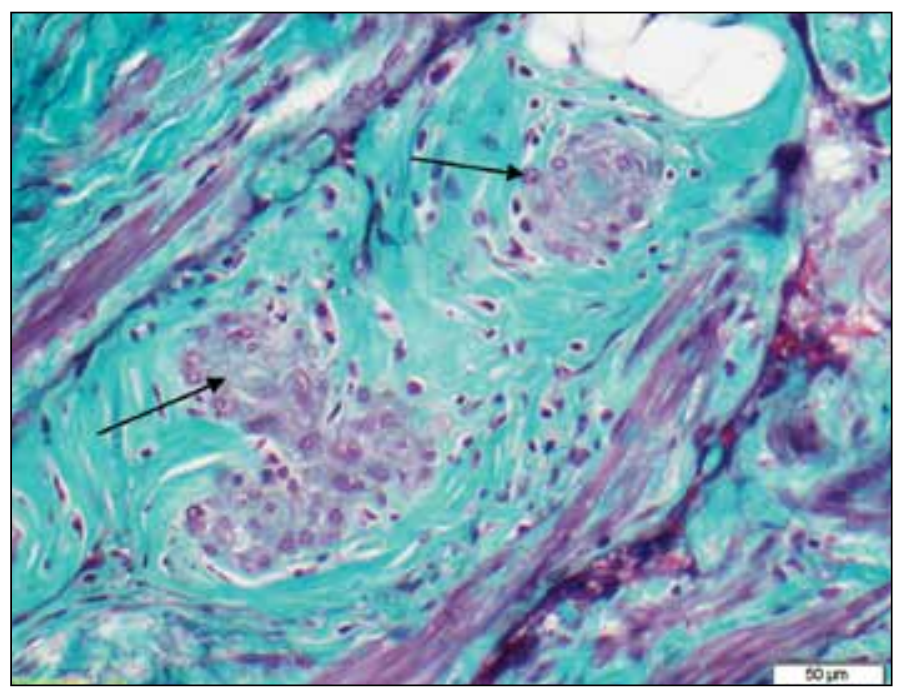

Fig. 4. Medial hypertrophy of small arteries with luminal occlusion(elastic trichrome stain, original magnification 100x).

3. Stone NN, Stock RG. Prostate brachytherapy in men with gland volume of $100 \mathrm{cc}$ or greater: Technique, cancer control, and morbidity. Brachytherapy 2013. pii: S1538-4721(13)00017-2. http://dx.doi. org/10.1016/i.brachy.2012.10.002

4. Lawton CA, Yan Y, Lee R, et al. Long-term results of an RTOG phase II Trial (00-19) of external-beam radiation therapy combined with permanent source brachytherapy for intermediate-risk clinically localized adenocarcinoma of the prostate. Int J Radiat Oncol Biol Phys 2012;82:e795-801. http://dx.doi. org/10.1016/i.irobp.2011.11.040

5. Theodorescu D, Gillenwater JY, Koutrouvelis PG. Prostatourethral-rectal fistula after prostate brachytherapy. Cancer 2000;89:2085-91. http://dx.doi.org/10.1002/1097-0142(20001115)89:10<2085::AlDCNCR8 $>3.0 .00 ; 2-0$

6. Shakespeare D, Mitchell DM, Carey BM, et al. Recto-urethral fistula following brachytherapy for localized prostate cancer. Colorectal Dis 2007;9:328-31.

7. Sur $M$, Sur $R$, Cooper $K$, et al. Morphologic alterations in esophageal squamous cell carcinoma after preoperative high dose rate intraluminal brachytherapy. Cancer 1996;77:2200-5. http://dx.doi. org/10.1002/(SICI) 1097-0142(19960601)77:11<2200::AID-CNCR3>3.0.C0;2-T

8. Mulhall JP, Yonover P, Sethi A, et al. Radiation exposure to the corporeal bodies during 3-dimensional conformal radiation therapy for prostate cancer. J Urol 2002;167:539-42.

Correspondence: Dr. John Kinahan, Division of Urology, Vancouver Island Health Authority, 1952 Bay Street Victoria, BC V8R 1]8, Victoria, BC; kinahan@shaw.co 